\title{
Collaborative Design Procedure for Supply Chain Process Integration Using UML
}

\author{
Francis E. Plonka, Mohammed S. Ahmed \\ Wayne State University, Detroit, USA \\ fplonka@mie.eng.wayne.edu, msal@roogna.eng.wayne.edu \\ Dan Carnahan \\ Rockwell Automation, USA \\ Dlcarnahan@Ra.Rockwell.Com
}

Keywords UML, Collaborative Design, Supply Chain, Process Integration

\begin{abstract}
The auto industry has embraced B2B e-commerce as a way of assembling their suppliers at one place so as to purchase parts and process equipment over the Internet. Suppliers who want to be successful in this environment will need to use Internet capabilities not only to quote projects but also to collaborate with other suppliers to execute the design and build activities at the quoted price, delivery and functionality requirements at an appropriate quality level. This will require interaction between product, process and tool engineers. Collaboration and interactions do not happen by accident. They require a common vocabulary and language along with explicit planning and management framework. The Unified Modelling Language (UML), is on its way to being adopted by the computer industry as the standard modelling language. This paper suggests that this object modelling language can be one component of this communication framework. In order to successfully use the Internet to accomplish these tasks, a framework/ procedure is needed to capture needed interaction and assignment of responsibilities that become the basis of the bid. These agreements can also be used to manage the interactions needed during design, construction and tryout stages. The proposed procedure utilizes a fundamental objectives tree to define the voice of the customer, the IDEF0 model language to define the life cycle of the design and construction activities and the UML to document and guide the needed interactions and communication. The process can also be used to manage the program in order to achieve the performance objectives. This paper describes the procedure and provides a case study for the design of a press material handling system. The equipment manufacturer relies on the die process and design functions as well as press manufacturer to interact at appropriate time to ensure successful operation of equipment.
\end{abstract}




\section{INTRODUCTION}

E-commerce is about building better relationships between customers, producers, suppliers and making communications that flow more efficiently while lowering cost. E-commerce can be divided into three categories; business-to-business, business-to-consumer and consumer-to-consumer [1]. When discussing e-commerce in a business-to-business framework, it is often associated with supply chain management and e-commerce's ability to improve the supply chain process.

Supply chain collaboration occurs when two or more companies share the responsibilities of exchanging common planning, management, execution and performance measurement information. Collaborative relationships transform how information is shared between companies and drive changes to the underlying business process, [2].

Supply chain strategies are undergoing tremendous changes. Outsourcing and partnering with other local or global enterprises are becoming more commonplace as companies seek to share the burden of demand for more complex products and more responsive services, [2]. These changes require greater collaboration and interaction with individual at diverse locations.

This paper will outline a framework for documenting the interactions and communication that take place between participants in the supply chain during a collaborative design process. Before we explain the methodology we will define three concepts that are being used in the paper. A more complete description of these concepts can be found in an article by the authors, [3].

\subsection{Function Objective Tree}

The Function Objective Tree (FOT) helps us to identify the Voice of the Customer that processes in the enterprise work to satisfy.

\subsection{IDEFO}

IDEF0 is a structured design and analysis technique that has been used extensively for the modelling of industrial and computer systems. The methodology allows the user to describe functions and activities and to decompose them into sub functions. The suite of IDEF tools, IDEFx, are used to model "as is" and "to be" scenarios, to create data models, to develop sequence logic and to simulate the throughput performance of the system. 


\subsection{UML}

UML provides a visual representation using object-modelling notation. The modelling of system elements using UML is recommended by the ISO TC 184/SC5/WG5 technical committee in their draft standard for Application Framework Requirements for Industrial Automation.

\section{METHODOLOGY - SUPPLY CHAIN COLLABORATIVE DESIGN PROCESS}

In this paper we are outlining a methodology that provides a systematic means of capturing and documenting the interaction that takes place in a supply chain collaborative design process. The aim is to outline the process and make it web-enabled so as to bring about a new dimension to ecommerce supply chain management. We name the methodology the Supply Chain Collaborative Design Process, (SCCD).

The SCCD identifies customer requirements by using the FOT. Once objectives are outlined and linked with the activities, IDEF0 is used to describe the life cycle of the design process. IDEF0 is then used to model each activity that makes up the process. The actors that are associated with the mechanisms are identified on the IDEF0 model. These actors and their interactions with the mechanisms are modeled as a Use Case model in UML. The final step is to develop various UML diagrams to document the process and also to outline the interactions that are needed.

\section{CASE STUDY: PRESS MATERIAL HANDLING SYSTEM}

The case study presented here is an extension of the research conducted under a NIST ATP grant to the Auto Body Consortium entitled the Near Zero Stamping, (NZS), Project. One NZS task outlined a process that documented the interaction and collaboration in developing a material handling system to move parts between stations in a progressive die. Next, we apply our methodology to capture and document the interaction that takes place between various parties while developing a material handling system for fabricating stamped sheet metal automotive parts.

In order to apply the SCCD process, we outline five basic customer requirements for an auto body and its associated parts. These primary customer requirements are set so as to achieve a near zero dimensional variation on the panels. These primary customer requirements are Precision Fit, Function, Finish, Fast (to market) and High Value to the customer. For 
each requirement a detailed FOT is developed to identify the role of the original equipment manufacturer (OEM) and their process equipment suppliers as well as the collaboration need to achieve it. Figure 4 shows the FOT for Precision Fit and Fast (to market).

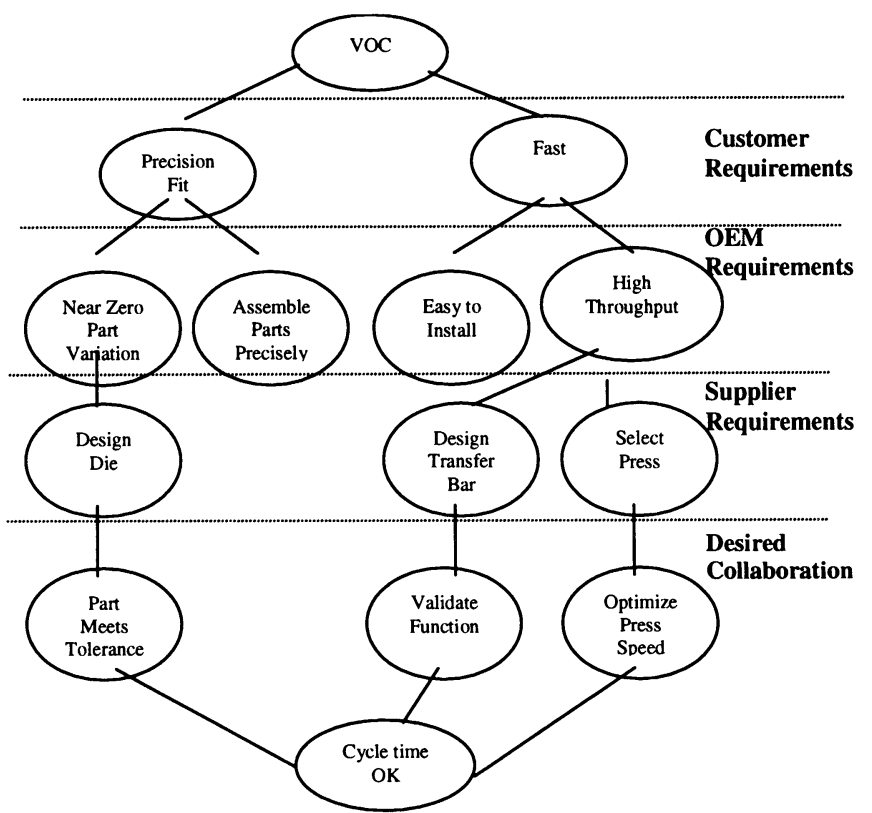

Figure 4 Function Objective Tree defining collaborative requirements

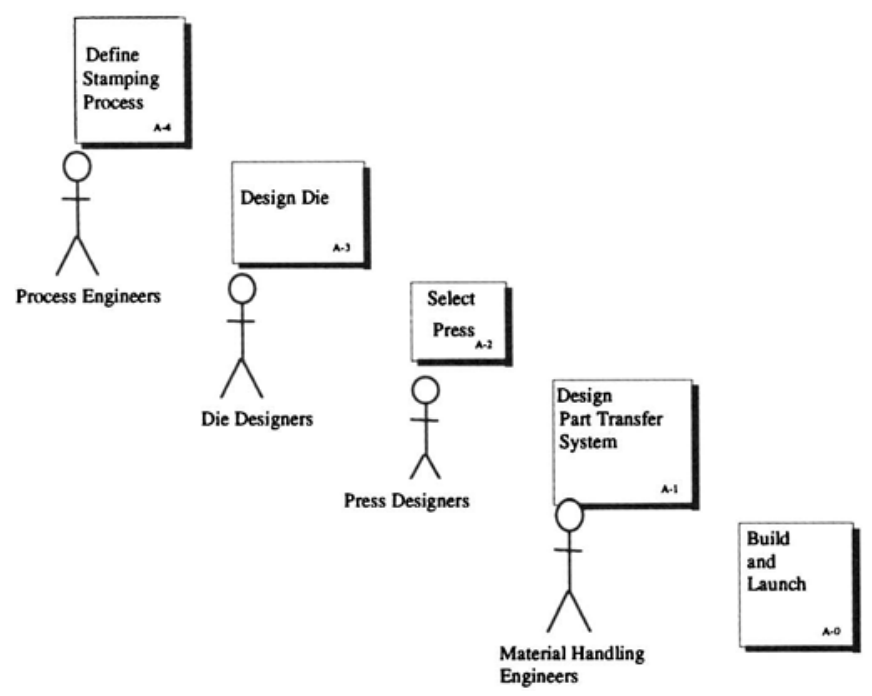

Figure 5 IDEFO Process for Press Material Handling System Design 


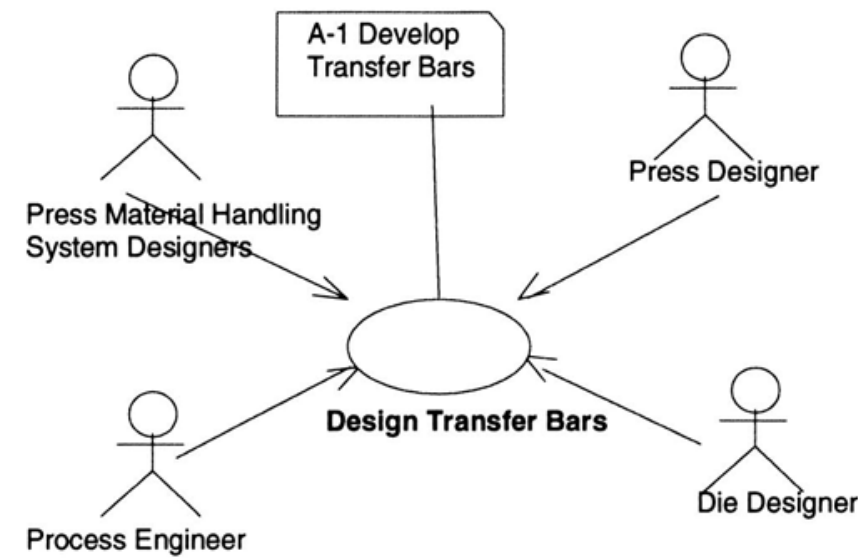

Figure 6 Use Case Diagram for "Develop Transfer Bar" activity

Using these requirements, activities are defined and a process can be outlined as shown in Figure 5. The IDEF0 model was developed based on interviews conducted with the following actors that make up the process. The interviews captured, in detail, the process of designing the press material handling system in a collaborative environment.

The next step is to develop a use case diagram using the UML as shown in figure 6. The diagram shows the interaction between use cases and their associated actors for the activity "Develop Transfer Bar" which is a subactivity of "Design Part Transfer System".

For each Use Case, a sequence diagram, is developed that outlines the sequence of operations for the system or the flow of functionality through the use case. A UML sequence diagram, depicted in Figure 7 shows how various actors exchange information to iterate through the design process. The diagram also shows the sequence of each transfer of information as well as, the process step performed by each actor. It is a complete representation of the collaboration that must take place defining when something must be done and when information must be transferred to support decision making for the task.

Focusing on one of the actors, we develop specific design requirements for various sub-systems based on the process needs. The requirements for each sub-system, such as the material handling, define the specific values for the various attributes for the sub-systems entities or "objects". Figure 8 illustrates the objects that define the task of the process engineer using a collaboration diagram. This provides a definition of his or her responsibilities in executing the process elements. The UML provides a rich set of diagrams that can adequately document individual roles and 
responsibilities as well as the interactions with others in carrying out each others job functions that ultimately satisfy the customer.

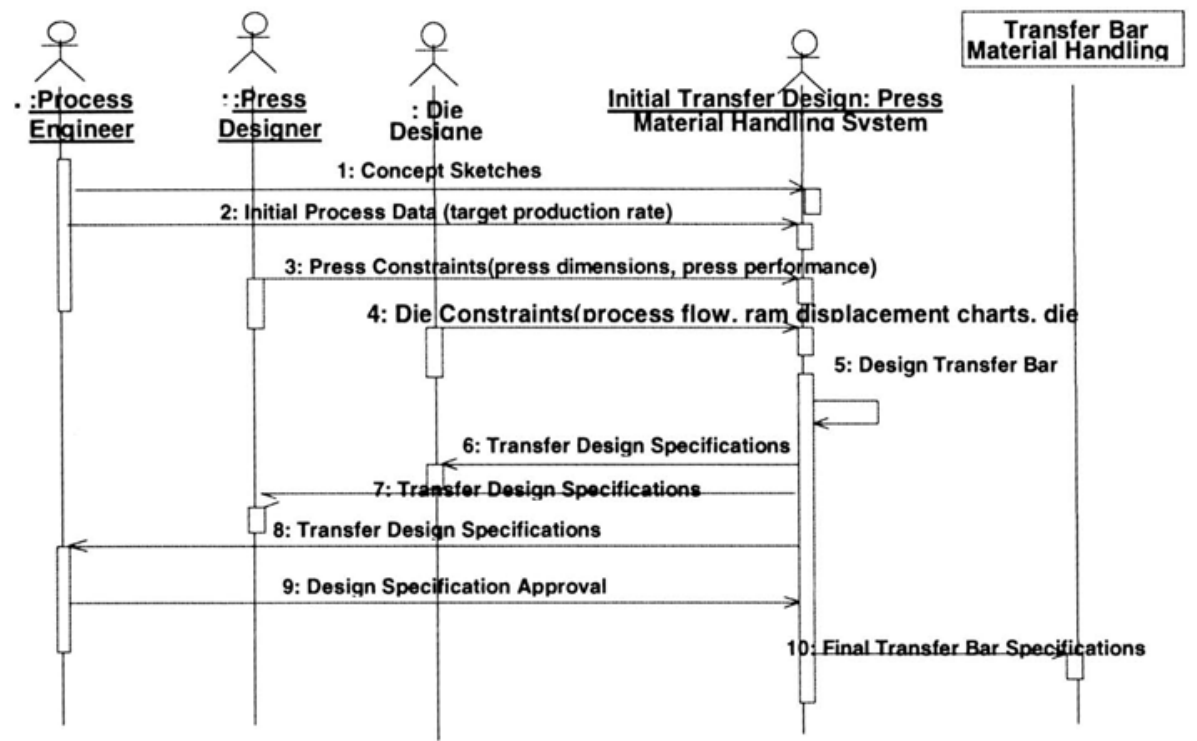

Figure 7 Sequence Diagram for "Develop Transfer Bars"

A collaboration diagram view of the requirements, as shown in figure 8, provides the first step in abstraction of the requirements to the various subsystem objects. While this figure is fairly simple, as more sub-system interaction become involved, the collaboration diagram provides a good view of the all of the interactions with the sub-systems.

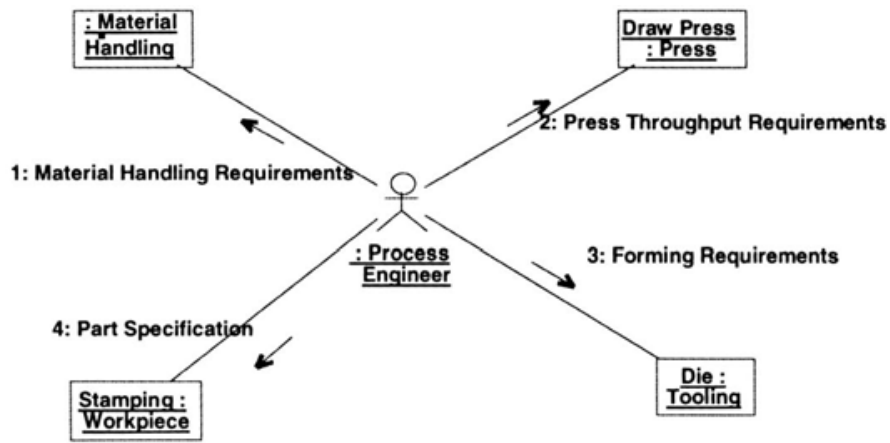

Figure 8 Collaboration Diagram for the Process Engineer

Figure 9 shows the roles and responsibilites of a process engineer using a sequence diagram. This provides a representation of when things must be done relative to each other. An indication of the time it takes to perform 
each specification and decision is shown as a small rectangular box on each vertical line.

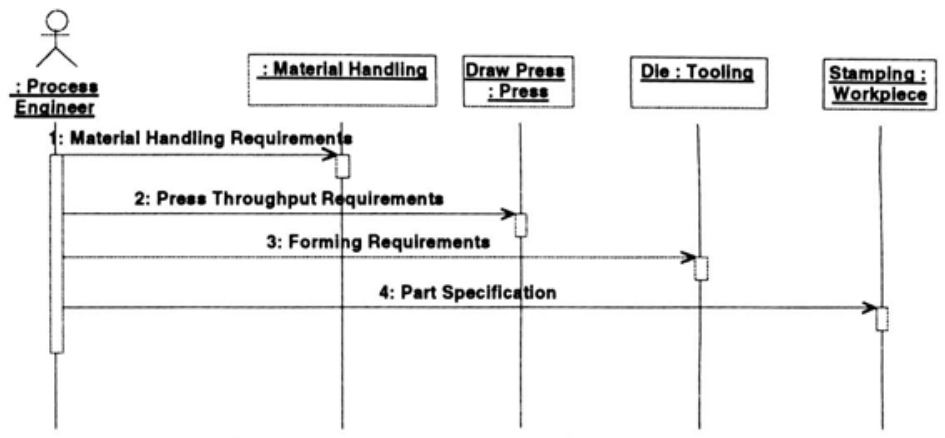

Figure 9 Sequence Diagram for the Process Engineer

Figure 10 is a representation of a class diagram that defines the end state desciption of each design artifact. It describes the information that must be defined and stored. The modelling language facilitates the design of screens, data bases and enabling programs that can support the web based collaboration. The class diagram depicts the various attributes and operations that the sub-system designer defines. By interacting with the users of the sub-systems (in this case study, the process engineer), the values of the attributes, and the timing of the operations can be specified based on the system design requirements. For instance, in the case of the material handling sub-system, the motion profile could be specified indicating a "cam profile," or it could be more generally specified using a mathematical expressions.

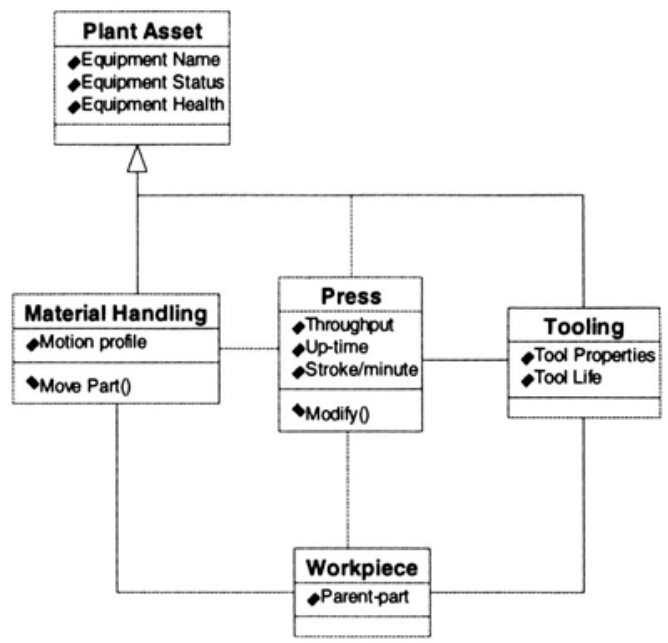

Figure 10 Class Diagram defining the end state description of each design artifact. 
Based on the UML diagram we can either implement the documented interaction or develop web-based tools that can facilitate collaboration. The web-based tool, Computer Aided Decision Support System, CADSS, was developed under the Near Zero Stamping Program that provides an effective interaction and communication between the Automobile Body supply chain participants.

\section{CONCLUSION}

The proposed methodology based on the Voice of the Customer, IDEF0 and UML permits the documentation of a process to be followed during the collaboration between individuals in a supply chain. The application of the procedure discussed in this paper involved the design, construction and installation of manufacturing equipment. A case study was provided regarding the design and construction of a material handling system for stamped sheet metal parts fabricated using a progressive die in a high-speed press.

The methodology provides several advantages for users. The first advantage permits the documentation of the interaction between individuals that can be used for collaborative design and training purposes. This demonstrates the power of the UML language in the training application domain. A second advantage of the methodology is that it provides interactive collaboration requirements for system developers of web based tools. A web-based system based on this methodology will enable and facilitate needed collaborative outcomes. This methodology can be easily extended and elaborated to develop web based tools to execute the desired collaborative interactions. A third advantage is that interactive information flows among the various actors in a collaborative environment are clearly delineated. The information exchange requirements provide the basis for developing simulations that can be used to validate the collaborative design process.

\section{REFERENCES}

[1] Robert Aaron; IEEE Communications Magazine September 1999, vol. 37, No 9; Electronic Commerce; Enablers and Implementation.

[2] Sherman Bai; NSF workshop on Supply Chain Management in Electronic Commerce, Department of Industrial and Systems Engineering University of Florida.

[3] Plonka, F. E., Carnahan, D., Ahmed, M. S.; Voice of the Customer ; An elaborative design technique for design synthesis and generation; Computer Technology Solutions for the Manufacturing Enterprise, SAE September 1999. 